\title{
Error Estimation for Indoor 802.11 Location Fingerprinting
}

\author{
Hendrik Lemelson ${ }^{1}$, Mikkel Baun Kjærgaard ${ }^{2}$, Rene Hansen $^{3}$, and Thomas King ${ }^{1}$ \\ 1 Department of Computer Science, University of Mannheim, Germany \\ \{lemelson,king\}@informatik.uni-mannheim.de \\ ${ }^{2}$ Department of Computer Science, University of Aarhus, Denmark \\ mikkelbk@cs.au.dk \\ 3 Department of Computer Science, University of Aalborg, Denmark \\ rhansen@cs.aau.dk
}

\begin{abstract}
. 802.11-based indoor positioning systems have been under research for quite some time now. However, despite the large attention this topic has gained, most of the research focused on the calculation of position estimates. In this paper, we go a step further and investigate how the position error that is inherent to 802.11-based positioning systems can be estimated. Knowing the position error is crucial for many applications that rely on position information: End users could be informed about the estimated position error to avoid frustration in case the system gives faulty position information. Service providers could adapt their delivered services based on the estimated position error to achieve a higher service quality. Finally, system operators could use the information to inspect whether a location system provides satisfactory positioning accuracy throughout the covered area. For position error estimation, we present four novel algorithms that take different features into account. Advantages of the combination of these four algorithms are explored by using a machine-learning approach. We evaluate our proposed algorithms in two different real-world deployments by using real-world data and emulation. The results show that our algorithms work independently of the environment and the positioning algorithm, and with an average precision for estimating the position error of up to 1.45 meters. The algorithms can - by adjusting parameters - realize different tradeoffs between underestimating and overestimating errors. Furthermore we comment on the algorithms' space and time complexity.
\end{abstract}

\section{Introduction}

Nowadays, powerful and light mobile devices, such as smart-phones and laptops, provide nomadic users with computing and networking services wherever they go. However, the device's usefulness can be highly leveraged by so-called location-based services (LBS): These services adapt their behavior proactively depending on the user's current location. A service that informs a user about the location of nearby friends and points of interest is a good example of an LBS.

Today, the most prominent source of location information is the Global Positioning System (GPS) [1]. GPS works well in outdoor open-sky environments but fails to deliver reliable location information where users spend most of their time: indoors and 
in so-called street canyons where tall buildings prevent users from getting a line-ofsight to GPS satellites [2]. Due to these drawbacks of GPS, in recent years a lot of effort has been spent on the development of alternative systems to estimate a user's position in GPS-free areas. However, current systems either use specialized equipment to work accurately in confined indoor environments (e.g., Ubisense ${ }^{1}$ ) or provide metropolitan-scale coverage at the expense of coarse positioning accuracy (e.g., Place Lab [3], RightSPOT [4]).

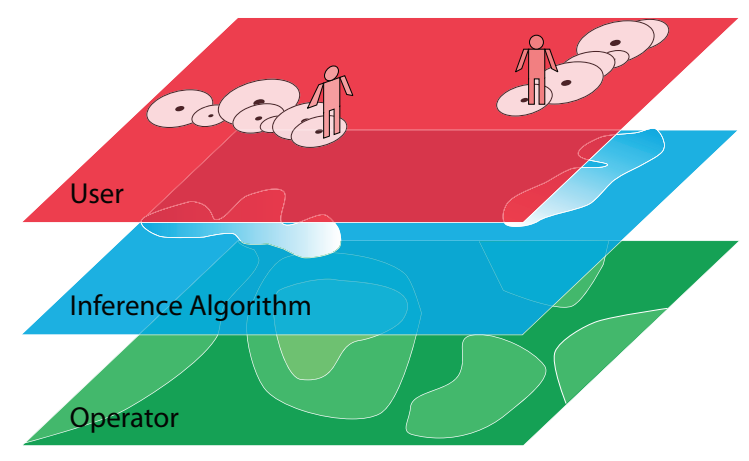

Fig. 1: The use of information about expected errors by users, inference algorithms and operators.

For three reasons, positioning based on 802.11 [5] offers a viable solution where other systems fail: Firstly, no specialized hardware is required, because, nowadays, almost all modern mobile devices are equipped with 802.11 network interfaces. Secondly, due to the proliferation of 802.11 wireless networks, radio signals from at least a few 802.11 access points can almost always be collected where people work and live in the developed world (e.g., [2] and [6]). Thirdly, 802.11 signals can be used to estimate a user's position indoors with an accuracy that is generally sufficient for most LBSs [7].

However, as with GPS, 802.11-based positioning systems comprise an inherent position error that is caused by radio propagation effects (e.g., reflection, diffraction, and scattering) and limits of the hardware (e.g., the measurement accuracy of sensors). Even worse, under sub-optimal conditions such as sparse coverage of access points or many moving obstacles that cause radio signals to fluctuate the accuracy of 802.11-based positioning systems tends to decrease.

GPS handles variations in the position accuracy by offering a measure called $D i$ lution Of Precision (DOP). Computed from several different system parameters, the DOP value indicates the level of position error that has to be expected for a given estimated position. Position error estimation is mainly valuable in three ways (see Figure 17: Firstly, the user can be notified that the estimated position might contain some position error. This helps him to make better use of the LBS [8] and also avoids him becoming frustrated because of many unexpected wrong positioning results. For instance,

\footnotetext{
${ }^{1}$ http://www.ubisense.net
} 
if a friend-finder service does not only show the estimated position of a friend but also the estimated position error (e.g., as a circle around the friend's position), a user might look around in the area indicated by the position error estimate in case he is not able to find his friend at the place where the service estimated him to be. Secondly, so-called inference algorithms utilize position estimates to derive additional information (e.g., activities or routes). Position error estimates allow the inference algorithms to assess the level of trust that these position estimates contain. As a result, the algorithms can give a higher priority to estimates with a low estimated error. This helps to deliver a higher service quality. Thirdly, operators of 802.11-based positioning systems can utilize position error estimates to optimize their system to the precision required by the application. For instance, an operator might add additional access points to the parts of the operation area that show position error estimates above a certain threshold.

In this paper, we propose several algorithms to estimate the expected position error of 802.11-based positioning systems. We focus on the static case of position estimation without any knowledge of spatial or temporal history and selected two well-studied systems proposed by Bahl et al. [9] and Haeberlen et al. [10] as positioning systems. These systems use a so-called location fingerprinting approach. Location fingerprinting consists of a two-stage mechanism: A training phase and a position determination phase. During the training phase, signal strength samples of nearby access points are gathered at reference spots in the operation area and stored together with their physical coordinates in a database: They are called fingerprints. In the position determination phase, the user's mobile device performs signal strength measurements at its (yet unknown) position and searches for similar patterns in the database. The closest match is selected, and its coordinates are returned as the position estimate.

We selected these two systems, as they represent the two main types of location fingerprinting algorithms, i.e., a probabilistic and a deterministic approach. They are easy to understand and therefore do not blur the view for the mechanisms used by our own error prediction algorithms. The two positioning systems differ in the way they process fingerprints and how they select the closest match. The system proposed by Haeberlen et al. builds signal strength distributions for each fingerprint based on the signal strength samples it contains. In the position determination phase, for each fingerprint a probability is calculated based on the currently collected signal strength samples. The probability calculation is done by utilizing Bayes' rule. The fingerprint with the highest probability is selected, and its position is returned as a position estimate. In contrast, the system proposed by Bahl et al. does not use distributions and probabilities. Instead, for each fingerprint the different signal strength samples are averaged. Based on these averages, the fingerprint that is closest to the average of signal strength samples collected during the position determination phase is selected. The calculation of the distances is done by applying the Euclidean distance.

We propose position error estimation algorithms that are able to predict the expected position error in advance, using only the collected training data, as well as algorithms that infer the expected position error from live measurements in the position determination phase.

We make the following contributions in this work: First of all, we propose novel algorithms for estimating the expected error of an indoor 802.11-based positioning sys- 
tem. Secondly, we provide a deep investigation by means of emulation to show that the proposed algorithms can estimate expected position errors with high accuracy. The emulation is based on collected real-world data and shows that our error estimation algorithms work independent of the environment and the positioning algorithm. Thirdly, we evaluate the idea of using a machine-learning algorithm to combine the proposed algorithms and to find an optimal combination of the proposed algorithms. Finally, we analyze the time and space consumption of the proposed algorithms.

The remainder of this paper is structured as follows: The work of other researchers related to our own findings is presented in Section 2 In Section 3, we introduce the proposed error estimation algorithms. This is followed by a description of the experimental setup and research methodology used to evaluate our error estimation algorithms in Section 4. Subsequently, the experimental results for the different algorithms alone as well as for a combination of algorithms are presented in Section 5 . In Section 6 we discuss the results of the evaluation. Finally, Section 7 concludes the paper.

\section{Related Work}

Some work that tries to understand the position errors for 802.11-based positioning systems already exists. However, the main difference compared to our work is that they try to form a priori analytical models in order to understand the position error in general e.g., to understand the relationship between the number of access points or the fingerprinting grid size and the positioning error. Our algorithms instead use information from actual fingerprints and position determination measurements to estimate the expected errors in an actual deployment area and at the time of use.

Analytical models have been proposed by Wallbaum [11], Kaemarungsi et al. [12], Krishnakumar et al. [13] and Youssef et al. [14]. They propose analytical models built from information such as access point placement, system parameters and floor layout. Using these models they analyze the general impact of access point placement, number of access points, grid size, signal variance, propagation constants, and floor layout on the position error. However, as commented earlier, these models are analytical tools for doing general analysis. Our algorithms go into the opposite direction, estimating expected errors at a specific site and at a specific point in time. However, it is an interesting future path of research to further analyze the relationship between algorithms for error estimation and analytical error models.

More related to our work are the methods for estimating outdoor GSM positioning errors proposed by Dearman et al. [8]. Motivated by a study on how to visualize expected GSM position errors they propose initial methods for how to estimate such information. They propose two methods for outdoor error estimation: A machine-learning method that uses a linear regression technique on basic signal strength features and a ground truth method that requires the collection of additional test data. The outdoor accuracy of these methods is up to 50 meters when considering the median accuracy. Their machine-learning method is similar to our effort of combining features, however, the used set of features is different. Their method is based on basic features such as signal strength variance. Our results show that for 802.11 such basic features can be outperformed by more advanced features such as the proposed fingerprint clustering 
algorithm. Furthermore, a machine-learning method might not be optimal because it will try to optimize the average precision. In several of our machine-learning experiments this led to a high percentage of either overestimations or underestimations for the expected position error.

\section{Error Estimation}

In this section, we propose our four novel algorithms for position error estimation. The following two algorithms can predict the expected positioning error in advance, using only the data collected in the training phase:
A. Fingerprint Clustering
B. Leave Out Fingerprint

The two other algorithms infer the expected position error from live measurements in the position determination phase:
C. Best Candidate Set
D. Signal Strength Variance

\subsection{Fingerprint Clustering}

The fingerprint clustering algorithm uses the idea, that inside structurally limited areas the signals received from an access point - despite inevitable small scale fading effects - tend to be within a certain range. For instance, the signal strength measurements collected in one single office room often cover only a quite limited range of the generally possible values. As in such a case all the fingerprints collected in this office room are very similar, a positioning algorithm will hardly be able to make an exact position estimation. Instead, it will probably select one of the other fingerprints collected somewhere else in the room.

If such an area of similar signal properties has large physical dimensions, we expect the position error to be larger because generally the number of similar fingerprints is higher and also their physical distribution space is larger. We exploit this behavior in the following way. Our novel algorithm lays out a grid of cells over the complete covered area of operation of the 802.11-based positioning system. Then the following steps are performed in order to find regions of similar signal properties:

1. Initially each grid cell represents a single cluster containing all the training samples collected inside the physical area of the cell.

2. After that, the algorithm randomly selects a cluster. By using a similarity measure, the algorithm then checks for the selected cluster whether the similarity to adjacent clusters lies above a given threshold. If this is the case for any adjacent cluster, the two clusters are merged into one new cluster.

3. The previous step is repeated until, for all remaining clusters, no further clusters can be merged.

4. As a next step, the set of clusters is checked for clusters that only comprise one single cell. If such a cluster is found, it is merged with its most similar adjacent cluster without considering the threshold. The reason for this procedure is that these single 
cell clusters most often are a result of very time and space constrained phenomena during the collection phase of the training data, and eliminating them helps the algorithm to deliver better and more stable results.

5. In the end, each remaining cluster represents a region of similar signal properties in the area of operation. The size and shape of the region is defined by the grid cells the cluster is comprised of. The estimated error for an estimated position is deduced from the size of the region the estimated position is located within.

An example for a region map resulting from the fingerprint clustering algorithm is given in Figure 2

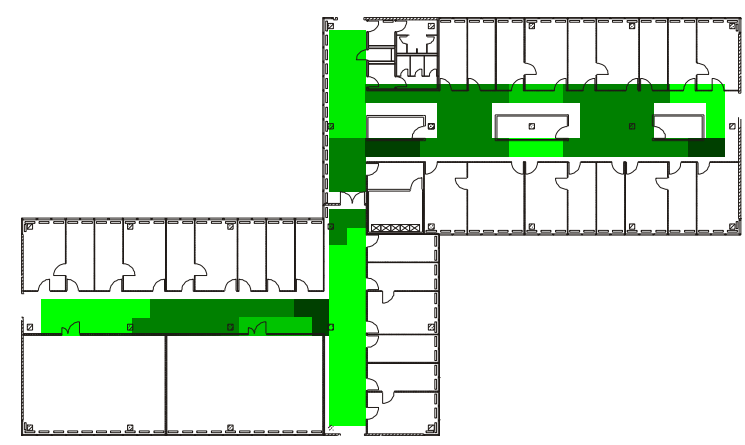

Fig. 2: Example for a region map created for the Mannheim testbed.

The similarity measure for a pair of clusters is calculated by comparing the distributions of signal strengths in the clusters as follows: For each access point of which signal strength measurements are contained in the samples of both clusters, and for each of the two clusters the mean and the standard deviation of the measured signal strengths are computed. These values are then used to create two normal distributions, each one representing the signal strength distribution of the specific access point and cluster. Afterwards, the intersection area of these two normal distributions is calculated (see Figure 3). This step is repeated for all access points until finally, the average size of all intersection areas is computed and interpreted as the similarity of the two clusters. Access points of which signal strength measurements are only contained in the samples of one cluster are not considered. Even though this could be easily done, e.g., by substracting a penalty from the computed similarity value, we decided to omit such a step. Due to the spatial neighbor property of the clusters that are pairwise considered, the case where there are major differences in the set of contained access points generally is very rare.

To figure out the influence of the similarity threshold on the performance of the algorithm, we conducted a pre-evaluation with varying settings for the threshold ranging from 0.4 to 0.6 . In general, a higher similarity threshold results in the regions becoming smaller. This helps to decrease the discrepancy between estimated and true error in cases where the correct region is chosen. But it also increases the rate of wrong region 

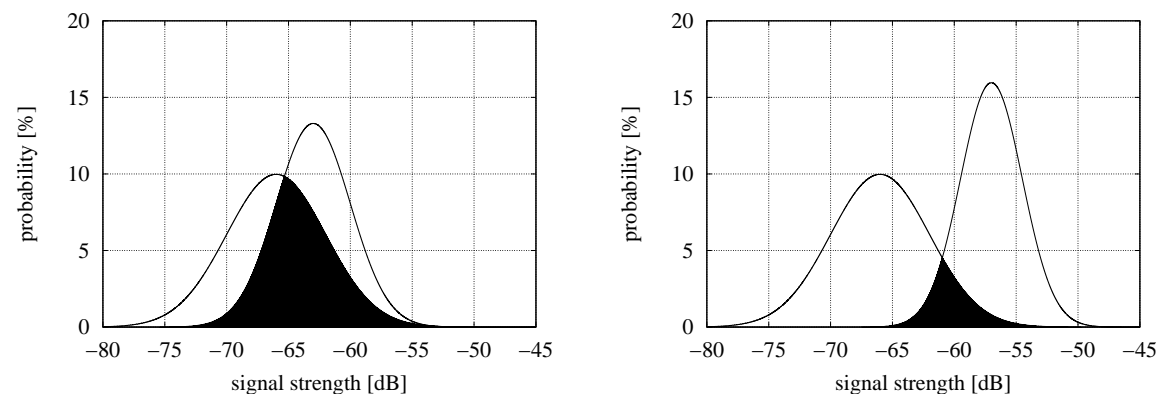

Fig. 3: Examples for the matching of signal strength distributions of one access point and adjacent clusters (good match on the left, bad match on the right hand side).

selections (also refer to Section 5.3). From the evaluated values, for our scenarios 0.51 performs best in terms of quantitative error estimation. Using this similarity threshold, the real and the estimated position are in the same region in $90 \%$ of all cases. Additionally, in half of the remaining cases, the real position is located in a cell directly adjacent to the selected region. Only in $5 \%$ of all cases, the real position is somewhere else. Therefore we have selected the value 0.51 as the similarity threshold for all further experiments.

\subsection{Leave Out Fingerprint}

The leave out fingerprint algorithm estimates errors by evaluating positioning performance using only the data collected during the training phase.

The performance evaluation uses an (n-1) cross evaluation with the constraint of considering only folds which contain data from a single fingerprint. This means that a positioning algorithm itself, for one current fingerprint, determines the closest match amongst all other fingerprints (the other (n-1) folds). The result is a good indicator of the error that can occur if the data collected during the position determination phase is noisy.

The position error result from this evaluation is used to compute an error map where each fingerprinted position is assigned an error estimate. In the position determination phase the position estimated by the positioning algorithm is looked up in the error map, and the error estimate stored for the corresponding position is returned.

For each fingerprinted position $p$, the error map contains an error estimate that is created by the following four steps.

1. Create a radio map using all fingerprints except the one for position $p$.

2. Run emulation using $m$ samples as test data taken randomly from the fingerprint for position $p$.

3. Calculate the observed errors for the position estimates from the emulation.

4. Calculate the error estimate for position $p$ as the average plus two times the standard deviation of all the observed $m$ errors. This method for computing the error estimate 
was selected because initial experiments showed that a conservative estimate gives the best results.

As a final step when all error estimates have been calculated, the error map is averaged to filter out local variations. This is performed by assigning a new estimate to each position. The new estimate is calculated as $66 \%$ of the original estimate and $33 \%$ as the average of the estimates for all neighboring fingerprints. We chose these parameters for the weights because they provide a good balance between the error estimates for the different fingerprint positions. In our evaluation this interpolation step increases the accuracy of the algorithm by several percent.

\subsection{Best Candidate Set}

When an 802.11-based positioning algorithm estimates a position, it generally returns only the position of the fingerprint that offers the best match to the data collected in the position determination phase. However, the best candidate set algorithm exploits the fact that positioning algorithms can return information about the second, third etc. best matches. The best candidate set algorithm uses the $n$ best estimates returned from a positioning algorithm to estimate the expected position error. The rationale for using the $n$ best estimates is based on the observation that positioning algorithms will often estimate a user to be at any of the nearby positions to his actual position. This is a result of the fact that adjacent fingerprinted positions will often exhibit highly overlapping signal strength properties while online samples similarly have sufficient signal strength variance to choose at random between close locations.

The best candidate set algorithm deduces an error estimate by computing the average distance between the best estimate and the next $(n-1)$ best estimates. The algorithm contains the following steps:

1. Form the set of the $n$ best estimates as outputted from an 802.11-based positioning algorithm.

2. Compute the distance between the position of the best estimate and all the other $(n-1)$ best estimates.

3. Return the average distance as the estimated error.

In addition to using the average distance between the best and the $(n-1)$ next best estimates, we also tried two other options to compute an error estimate from the best candidate set. The first option was to use the maximum distance between the best estimate and any of the $(n-1)$ next best estimates. The second option was to compute the maximum distance between any two of the $n$ best estimates. Both options led to more conservative error estimates but they were also more sensitive to large position estimation errors and increasing $n$. In effect, both options produced a higher proportion of large over-estimations when the performance of the positioning algorithm degraded or when the size of the best candidate set was increased. The average-distance approach in contrast handled these situations better. Using the average distance, it was found that $n$ set to three gave the best overall performance. Higher values of $n$ made the error estimates more conservative while gradually decreasing performance due to the inclusion of more faraway positions. 


\subsection{Signal Strength Variance}

Like most 802.11-based positioning systems, the systems selected for this paper are error-prone to signal strength variations, because the position estimation process depends on probabilities or average values calculated on the signal strength samples.

The reasons for the variation of signal strength samples are two-fold: Firstly, smallscale effects and multi-path radio propagation cause signal strength values to vary significantly if the mobile device moves within the wavelength (for 802.11 the wavelength is 12.5 centimeters). So, both during the training as well as during the position determination phase, even if the mobile device is supposed to be static, small movements can have a big impact on the measured signal strength. Secondly, movements in the surrounding of the mobile device in question (e.g., persons roaming around or doors being automatically opened or closed) cause radio signals to travel different ways which in consequence causes the signal strength to fluctuate.

If the variance between different signal strength samples is high the probability that the actual closest fingerprint is selected is rather small, and a fingerprint far away might reach a higher probability. The same is true for the average calculation, because the average value might differ if the variance between different signal strength samples is high. Therefore an increase in variance also increases the chance of position errors.

The idea of the signal strength variance algorithm is to estimate the position error based on the signal strength variance of the samples that are used in the position determination phase to compute a position estimate. The algorithm can be described as follows:

1. For each access point that is part of the signal strength samples, find the largest signal strength value (in $\mathrm{dB}$ ).

2. Based on the largest signal strength value that is specific for a certain access point, subtract this value from all signal strength samples that are available for this given access point.

3. For each access point calculate the signal strength variance for the calculated values.

4. Average the signal strength variance values calculated for each access point to get an overall variance value. This variance value can be perceived as an indicator of the expected position error.

We also tried several other ways to calculate an overall signal strength variance value. However, this did not lead to an algorithm with better results than achieved with the algorithm presented above.

\subsection{Random Value}

As a baseline and to make the results of our own algorithms better comparable, we also implemented a simple random error estimation algorithm. This algorithm returns, independent of the supplied data, uniformly distributed random error estimates between 0 and ten meters. 


\section{Experimental Setup and Methodology}

In this section, we describe the experimental setup and the measurement methodology used to evaluate the proposed algorithms.

\subsection{Local Test Environments}

We deployed our 802.11-based positioning systems in two different environments: On the second floor of the Hopper building on the campus of the University of Aarhus and on the second floor of the office building A5,B on the campus of the University of Mannheim.

The former one is a newly built office building consisting of many offices and a long hall (see Figure 4a). The area is covered by 23 access points from different vendors; only five of these access points can be detected in at least half of the measurements. Nine faroff access points are even only detectable in less than ten percent of all measurements. The average number of access points contained in the fingerprints for this environment is 6.9 while the average number contained in the samples used for positioning is 4.8 . The 802.11-based positioning system covers an area of about 56 meters times 13 meters.

The latter environment is also situated in an office building and consists of many offices and three longer hallways (see Figure $4 \mathrm{~b}$ ). The area is covered by 25 access points in total although our data shows that most of the access points only cover parts of the operation area. In fact, only two access points cover the operation area completely. On average, 14.7 access points are contained in each fingerprint for this environment and 10.5 access points are contained in each set of measurements used for positioning during the position determination phase. The operation area is nearly 57 meters wide and 32 meters long.

\subsection{Hardware and Software Setup}

As a client, we used a Lucent Orinoco Silver PCMCIA network card supporting 802.11b. This card was plugged into an IBM Thinkpad R51 laptop computer. To collect signal strength samples, we used our own set of tools [15].

\subsection{Data Collection}

For both environments we applied a grid of reference spots with a grid spacing of 1.5 meters to the operation areas. For the Hopper building this resulted in 225 reference spots while the Mannheim environment comprises 130 reference spots (see the black markers in Figures $4 \mathrm{a}$ and $4 \mathrm{~b}$ ). For the former environment 120 signal strength measurements and for the latter one 110 signal strength measurements were subsequently collected at each reference spot.

For the position determination phase, we then selected 14 spots in the Hopper building and 46 spots in the A5-B building. At each of these spots, we collected 110 signal strength samples as well. In Figure $4 \mathrm{a}$ and Figure $4 \mathrm{~b}$, these spots are marked by light grey dots. 


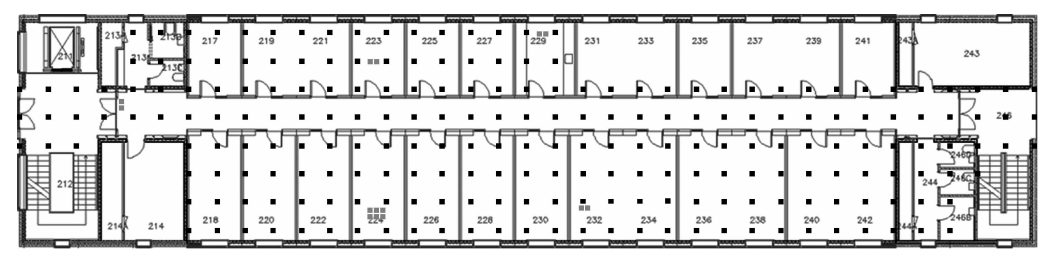

(a) The second floor of the Hopper building.

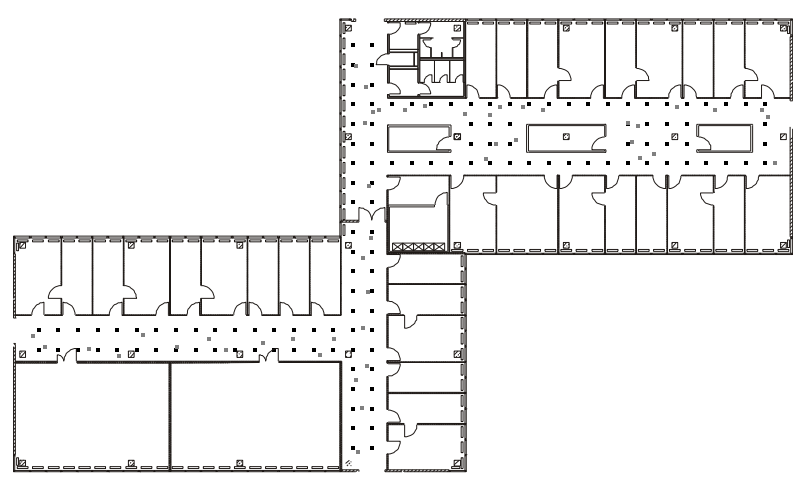

(b) The second floor of the A5-B building.

Fig. 4: The two test environments. The reference spots are marked in black and the test spots are depicted in light grey.

\subsection{Metrics}

Regarding the metric used to compare our error estimation algorithms, we use the error distance difference. For each position estimate, we compute the physical distance between the real and the estimated position (the real error) and then substract the result from the value that our error estimation algorithms return as the expected error. By using the error distance difference, it is e.g., easy for us to analyze whether our algorithms tend to estimate the position error too high or rather tend to estimate it too low.

\subsection{Experimental Methodology}

We performed an intense evaluation to analyze the properties of our four error estimation algorithms. To keep the results comparable, we examined the four algorithms in parallel. To achieve this, we used our suite of positioning-related tools [15] and modified it to suit our special needs.

The basic experiment consists of the following steps: At first, one of the two used positioning algorithms is initialized with 25 randomly selected samples per reference spot to build up its fingerprint database. Subsequently, our four different error estimation algorithms are initialized as well. Those that make use of the training data are additionally provided with exactly the same data as the positioning algorithm itself. The same is then done with the data for the position determination phase. At first, the 
positioning algorithm - for each test position - is given five online samples to estimate a position. Afterwards, the same samples used for the position determination as well as also the estimated position are provided to our error estimation algorithms. Each of the algorithms then solely computes an error estimate which is recorded and stored for later reference.

The values used for the number of training and position determination samples were chosen according to [7]. To achieve statistically stable results, we repeated our basic experiment 100 times. We ran our basic experiment by using the positioning algorithm proposed by Bahl et al. [9] and the one proposed by Haeberlen at el. [10] on data collected from both of our test environments.

As listed in Table 1 , the position accuracy of the positioning algorithms by Bahl et al. [9] and Haeberlen at el. [10] is quite different when considering the data for each of our two test environments. The main reason being the number of access points, the properties of the environments and the performance of the positioning algorithms themselves.

Table 1: Position accuracy for positioning algorithms and test environments.

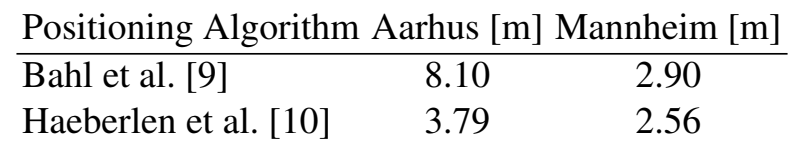

\section{Experimental Results}

In this section, we present our evaluation results focusing on the results for the more accurate Haeberlen et al. positioning algorithm.

\subsection{Estimation Accuracy}

The proposed error estimation algorithms show noticeable differences in their single performances as well as in their reactions to different environmental conditions and to the used positioning algorithms.

Overall, for the Aarhus dataset and with the Haeberlen et al. positioning algorithm the fingerprint clustering approach delivers the best results (e.g. 1.13 meters at the median) even though the second best algorithm, the best candidates set, is quite close regarding the $95 t h$ percentile (see Figure $5 \mathrm{a}$ ).

Looking at the Mannheim dataset, the situation has changed. Considering the 25th, $50 t h$, and $75 t h$ percentiles, the best candidates set algorithm now takes the lead with 1.10 meters at the median (see Figure 5b). This impression is also confirmed when taking a look at the average values for the error distance difference of the four algorithms and the two environments (see Table 2). Here as well, the best candidates set algorithm performs best for the Mannheim dataset at 1.45 meters while the fingerprint clustering algorithm is ahead for the Aarhus dataset at 2.24 meters. 


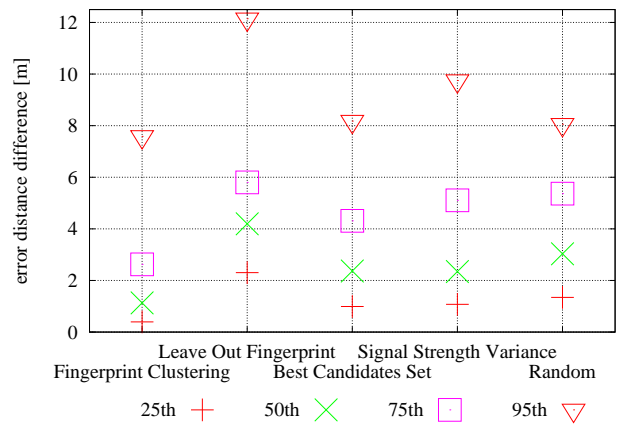

(a) Aarhus dataset

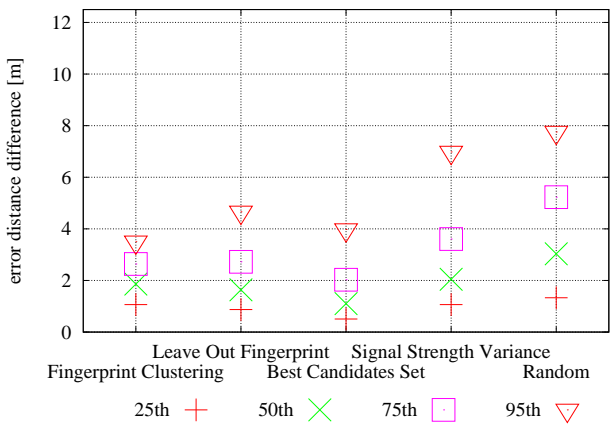

(b) Mannheim dataset

Fig. 5: Overview of the performance of the different algorithms for the Aarhus as well as for the Mannheim dataset using the positioning algorithm by Haeberlen et al.

The reason for these differences are the different properties of the datasets. For example, as listed in Section 4.5 the average positioning error for the Mannheim dataset is noticeably lower mainly due to the on average higher number of access points available. As the fingerprint clustering algorithm tends to make very conservative error estimations, this leads - compared to the rather optimistic estimations of the best candidates set algorithm - to the just introduced results.

The opposite is true for the Aarhus dataset. Here, due to the generally larger errors of the positioning algorithms, the fingerprint clustering algorithm can deliver the best results while the other error estimation algorithms have difficulties especially with estimating outlying errors.

For the results with the positioning algorithm by Bahl et al. the error estimation algorithms again perform best on the Mannheim data set. However, for this positioning algorithm the fingerprint clustering algorithm has the best performance on both data sets with an average accuracy of 2.03 meters for the Mannheim data and 6.56 meters for the Aarhus data. The reason that the result with the Aarhus data set is several times worse is that the accuracy of the error estimation is impacted by the low average position accuracy of 8.10 meters in this case. However, when comparing the decrease in position accuracy and error estimation accuracy it can be noted that they are on the same scale.

\subsection{Combining Algorithms}

In addition to evaluating the accuracy of each algorithm we have investigated if accuracy can be improved by combining the algorithms using a machine-learning algorithm. We used the Weka machine-learning toolkit [16] for our experiments. In order to feed the output of our evaluations to Weka, we implemented a small Java program that reads the evaluation results for the proposed error estimation algorithms and outputs an ARFF file for the Weka tool. Before finally selecting a machine-learning method to use for combining the results, we experimented with different methods, and concluded that the 
Table 2: Average error distance difference for the Aarhus and the Mannheim dataset using the positioning algorithm by Haeberlen et al.

\begin{tabular}{lcccc} 
& \multicolumn{2}{c}{$\begin{array}{c}\text { Aarhus Dataset } \\
\text { Algorithm }\end{array}$} & $\begin{array}{c}\text { Mannheim Dataset } \\
\text { Avg. Error [m] }\end{array}$ Std. Dev. [m] & Avg. Error [m] Std. Dev. [m] \\
\hline Fingerprint Clustering & 2.24 & 2.91 & 1.90 & 1.09 \\
Leave Out Fingerprint & 4.68 & 3.53 & 1.95 & 1.47 \\
Best Candidates Set & 3.06 & 2.61 & 1.45 & 1.26 \\
Signal Strength Variance & 3.92 & 5.08 & 2.69 & 2.45 \\
Random & 3.58 & 2.84 & 3.43 & 2.39
\end{tabular}

Least Median Squared Linear Regression method was most appropriate. This method uses regression to learn weights for a linear model. The weights are learned from a training set (e.g. either the Aarhus or Mannheim data set) and then the learned model is tested using a test set (e.g. the set that was not used for training).

The results of different combinations of the proposed algorithms as well as test and training data are listed in Table 3 When combining error estimates of all the algorithms and testing with the Mannheim data an average result of 1.37 meters is achieved which is a marginal improvement over the 1.45 meters for the Best Candidate Set algorithm alone. When testing with Aarhus data the result is 2.40 meters which is a bit worse than 2.24 meters for the fingerprint clustering algorithm alone. The table also lists results when only the output of the best candidate set and fingerprint clustering algorithms are combined. These results are only a few centimeters better on average than when combining all algorithms. Given the small improvement, using a single algorithm seems like a better option than combining several algorithms, especially when considering the additional system complexity and increased computational requirements for running several algorithms in parallel and then later combining their output.

Table 3: Results for different combinations of the proposed algorithms and test and training data for the positioning algorithm by Haeberlen et al.

\begin{tabular}{llll}
\multicolumn{2}{l}{ Algorithms } & Training Dataset & Test Dataset Avg. Error $[\mathrm{m}]$ \\
\hline All & Aarhus & Mannheim & 1.37 \\
Two Best & Aarhus & Mannheim & 1.35 \\
All & Mannheim & Aarhus & 2.40 \\
Two Best & Mannheim & Aarhus & 2.36
\end{tabular}

\subsection{Over Estimation versus Under Estimation}

Some applications might have preferences with respect to overestimation or underestimation of position errors. For instance, an application that has to alert a user in case the estimated error is above a threshold might prefer overestimation in order to avoid to not alert the user (false negatives). On the other hand, an application that sends the 
user information about shops in his proximity might prefer an underestimation of the error to avoid annoying the user with too many messages. In Figure 6 the error distance difference distributions are depicted for the two most promising algorithms. The positioning system proposed by Haerberlen et al. is used and data for both test environments is displayed. In these distributions underestimations result in negative values while overestimations lead to positive values. Our results show that the best candidates set algorithm has a tendency to produce a higher amount of large overestimations. Also, when comparing the results for the Aarhus and Mannheim datasets, the distributions reveal that both algorithms produce a higher amount of large under- and over-estimates for the less accurate Aarhus dataset.

Our proposed algorithms offer the possibility to adjust the error estimation performance to favour either under- or overestimation with algorithm-specific parameters. This comes at the expense of reliability, though. For example, for the best candidates set algorithm, the size of the set can be varied. When considering more candidates in our evaluations, the number of errors that are underestimated decreases. But at the same time, the amount of overestimation increases because more, possibly physically far distinct positions are taken into account. The same is true for the fingerprint clustering algorithm. When adjusting the similarity threshold for the merging of clusters, the size of the resulting regions increases. This also increases the probability of the estimated and real position being located in the same region. But at the same time it also increases the average error distance difference in our evaluations. Therefore, here again, we have a tradeoff between accuracy and reliability.

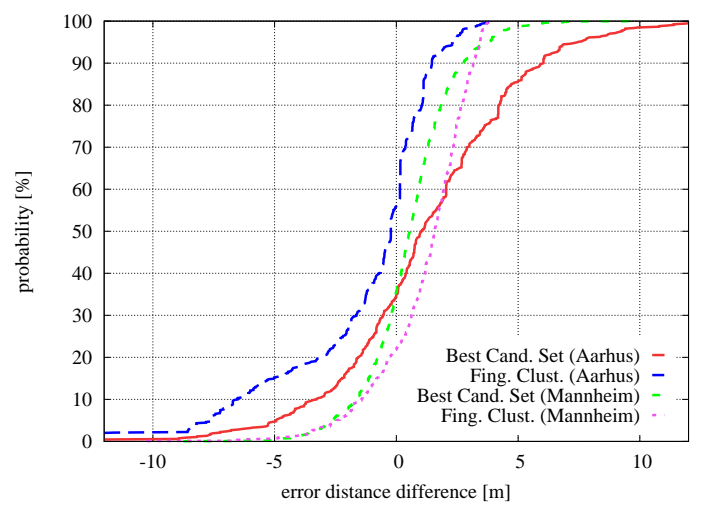

Fig. 6: Distributions of the difference between estimated and real error

\section{Discussion}

This section discusses the space and time complexity of the proposed algorithms, the influence of the number of position determination samples and the number of access points on the error estimation, and the GPS approach for the dilution of precision. 


\subsection{Space and Time Complexity}

Considering the space and time complexity of our algorithms, we have to distinguish between those operating only on the training data and those also taking the measurements during the position determination phase into account.

For the former ones, computational complexity is rather not critical as the algorithms need to be run on the training data only once. This can be done on powerful systems in advance of the deployment. The opposite is true for the space complexity. As the result of the algorithms, depending on the setup of the positioning system, might be stored and processed on the mobile device, here the amount of data is important.

For the latter ones, both space and time complexity are important. For each single position determination, a computation based on the current set of measurements as well as the training data takes place. Considering that this might as well take place on a mobile device, the algorithms have to be economical regarding CPU time and memory consumption. However, it turns out that both the fingerprint clustering and the best candidate set algorithms are light-weight in terms of computations and space use, please refer to Table 4 for the details.

Table 4: Comparison of the space and time complexity for the proposed algorithms, where $\mathrm{c}$ is the number of initial cells, $\mathrm{n}$ is the number of fingerprints, $\mathrm{p}$ is the time complexity of the positioning algorithm, a is the number of access points in the online samples, $\mathrm{b}$ is the size of the best candidate set and $\mathrm{h}$ is the number of stored samples.

\begin{tabular}{lcccc} 
& \multicolumn{1}{c}{ Fingerprint Clustering Leave-Out Fingerprint Best Candidate Set Signal Strength Variance } \\
\hline Time complexity & $O(c)$ & $O(n * p)$ & $O(b)$ & $O(a * h)$ \\
Space complexity & $O(c)$ & $O(n)$ & $O(b)$ & $O(a * h)$
\end{tabular}

\subsection{Number of Position Determination Samples and Number of Access Points}

From experimental results King et al. [7] conclude that the 802.11 position error is mainly determined by the number of access points and the number of signal strength samples used in the position determination phase. In this work, we do not present any algorithm that takes this fact explicitly into account. However, the previously presented algorithms indirectly adhere to this fact. For instance, the fingerprint clustering algorithm (see Section 3.1p when comparing similarity between clusters iterates over the set of access points and by such explicitly handles the number of access points. The number of position determination samples is explicitly covered by the signal strength variance algorithm (see Section 3.4) as the variance value stabilizes if the number of signal strength samples increases. However, it would be an interesting path of future work to analyze if the proposed algorithms could be extended to more explicitly use such results. 


\subsection{Dilution of Precision}

In addition to the proposed algorithms, we have also implemented an algorithm that computes a GPS-like DOP value based on the geometry of access points. The algorithm uses the equations for DOP values described in Borre et al. [17]. However, a DOP value is not an error estimate in meters but a factor that has to be combined with an error estimate. Therefore we have evaluated if the accuracy of the proposed algorithms can be improved by combining their output with a DOP value by multiplication. However, for all of the algorithms the combination with a DOP value strongly reduces their accuracy. This suggests that GPS-like DOP values do not seem to be a promising error estimation approach for indoor 802.11-based positioning. The main reason for that is that the complex indoor propagation environment makes the geometry of the access points less important for the positioning accuracy than it is in the case of outdoor satellite-based positioning. This result is consistent with the analytical results for the impact of the geometry of access points on the position accuracy presented by Wallbaum et al. [11].

\section{Conclusion and Further Work}

In this paper, we have introduced four novel algorithms to estimate the position error of 802.11-based positioning systems. Our algorithms exploit several different features to fulfill their task. We have proposed error estimation algorithms that only use training data as well as algorithms that take only the data available during the position determination phase into account.

All our algorithms deliver good results for estimating the position error both for different environments and for the different positioning algorithms. The fingerprint clustering algorithm and the best candidates set algorithm though perform especially well. With either of these two algorithms it is possible to estimate the position error of an 802.11-based positioning system to a high degree of accuracy and with sufficient reliability. This is in contrast to the geometry-based algorithms used for GPS which fail to work for 802.11-based positioning systems.

We have evaluated if using a machine learning technique to combine several of the proposed algorithms can improve the precision of the error estimates. In our case though, the gain is very small or the results even worsen. We therefore suggest not to use any combination of the different algorithms but rather use one algorithm alone. Looking at the targeted use of the algorithms on mobile devices, this suggestion makes even more sense as the use of multiple algorithms - even though each single one economical in terms of processing and storage demands - would waste precious resources.

This work opens up several paths of future work. Firstly, to analyze if the proposed algorithms could be extended to more explicitly take information such as the number of position determination samples into account. Secondly, to further explore the relationship between algorithms for error estimation and analytical error models. Thirdly, the evaluated positioning algorithms were for reasons of analyzability not extended with advanced methods such as tracking. Therefore, a further path of future research will be to analyze how to optimize our algorithms when applying them to a positioning algorithm that uses e.g., tracking. 


\section{References}

1. Kaplan, E., Hegarty, C., eds.: Understanding GPS: Principles and Applications. Second edn. Artech House Incorporated (December 2005)

2. LaMarca, A., Chawathe, Y., Consolvo, S., Hightower, J., Smith, I., Scott, J., Sohn, T., Howard, J., Hughes, J., Potter, F., Tabert, J., Powledge, P., Borriello, G., Schilit, B.: Place Lab: Device Positioning Using Radio Beacons in the Wild. In: Proceedings of the Third International Conference on Pervasive Computing. (2005)

3. Cheng, Y.C., Chawathe, Y., LaMarca, A., Krumm, J.: Accuracy Characterization for Metropolitan-scale Wi-Fi Localization. In: Proceedings of the Third ACM International Conference on Mobile Systems, Applications, and Services. (2005)

4. Krumm, J., Cermak, G., Horvitz, E.: RightSPOT: A Novel Sense of Location for a Smart Personal Object. In: Proceedings of the 5th International Conference on Ubiquitous Computing. (2003)

5. Institute for Electrical and Electronics Engineers, Inc.: ANSI/IEEE Standard 802.11: Wireless LAN Medium Access Control (MAC) and Physical Layer (PHY) Specifications. Website: http://standards.ieee.org/getieee802/(1999)

6. Bychkovsky, V., Hull, B., Miu, A., Balakrishnan, H., Madden, S.: A Measurement Study of Vehicular Internet Access Using In Situ Wi-Fi Networks. In: Proceedings of the 12th International Conference on Mobile Computing and Networking. (2006)

7. King, T., Haenselmann, T., Effelsberg, W.: Deployment, Calibration, and Measurement Factors for Position Errors in 802.11-based Indoor Positioning Systems. In: Proceedings of the Third International Symposium on Location- and Context-Awareness (LoCA). (2007)

8. Dearman, D., Varshavsky, A., de Lara, E., Truong, K.N.: An exploration of location error estimation. In: Procedings of the Nineth Interational Conference on Ubiquitous Computing. (2007)

9. Bahl, P., Padmanabhan, V.N.: RADAR: An In-Building RF-based User Location and Tracking System. In: Proceedings of the 19th Annual Joint Conference of the IEEE Computer and Communications Societies. (2000)

10. Haeberlen, A., Flannery, E., Ladd, A.M., Rudys, A., Wallach, D.S., Kavraki, L.E.: Practical Robust Localization over Large-Scale 802.11 Wireless Networks. In: Proceedings of the Tenth ACM International Conference on Mobile Computing and Networking. (2004)

11. Wallbaum, M.: A priori error estimates for wireless local area network positioning systems. Pervasive Mobile Computing 3 (2007) 560-580

12. Kaemarungsi, K., Krishnamurthy, P.: Modeling of indoor positioning systems based on location fingerprinting. In: Proceedings of the 23th Annual Joint Conference of the IEEE Computer and Communications Societies. (2004)

13. Krishnakumar, A.S., Krishnan, P.: On the accuracy of signal strength-based estimation techniques. In: Proceedings of the 24th Annual Joint Conference of the IEEE Computer and Communications Societies. (2005)

14. Youssef, M., Agrawala, A.: On the optimality of wlan location determination systems. In: In Proceedings of the Communication Networks and Distributed Systems Modeling and Simulation Conference. (2004)

15. King, T., Butter, T., Lemelson, H., Haenselmann, T., Effelsberg, W.: Loc $\{$ lib,trace,eva,ana $\}$ : Research Tools for 802.11-based Positioning Systems. In: Proceddints of the ACM WiNTECH. (2007)

16. Witten, I.H., Frank, E.: Data Mining: Practical Machine Learning Tools and Techniques, Second Edition (Morgan Kaufmann Series in Data Management Systems). Morgan Kaufmann (2005)

17. Borre, K., Akos, D., Bertelsen, N., Rinder, P., Jensen, S.: A Software-Defined GPS and Galileo Receiver. Birkhuser (2007) 